\title{
Water clustering in polychloroprene
}

\author{
Pierre Yves LE GAC ${ }^{a,}{ }^{*}$, Gérard Roux ${ }^{b}$, Peter Davies $^{a}$, Bruno Fayolle ${ }^{c}$, Jacques Verdu ${ }^{c}$
}

\author{
a IFREMER Centre de Bretagne, Marine Structures Laboratory, BP70, 29280 PLOUZANE, France \\ b Thales Underwater Systems, TUS, route des Dolines, BP 157, 06903 Sophia-Antipolis Cedex, France \\ ${ }^{c}$ PIMM, Arts et Métiers ParisTech, 151 Bd de l'Hôpital, F-75013 PARIS
}

*: Corresponding author : Pierre Yves Le Gac, tel.: +(33)298224885 ; fax: +(33)298224535;

email address : pierre.yves.le.gac@ifremer.fr

\begin{abstract}
:
Water sorption has been studied gravimetrically for polychloroprene rubber samples, first at a fixed hygrometric ratio $(98 \% \mathrm{HR})$ and several temperatures $\left(25,40,60\right.$ and $\left.80^{\circ} \mathrm{C}\right)$ for samples of 1.9 and $3.8 \mathrm{~mm}$ thickness (Constant Temperature and Hygrometry, CTH experiments), then at fixed temperature $\left(40^{\circ} \mathrm{C}\right)$ and several hygrometric ratios ranging from 0 to $95 \% \mathrm{HR}$ on samples of $0.1 \mathrm{~mm}$ thickness (DVS experiments). CTH experiments reveal an abnormal sorption behavior: after an apparently fickian transient period, the water absorption continues at almost constant rate, no equilibrium is observed after more than 2500 hours, whatever the temperature. DVS experiments reveal a very low Henry's solubility but the formation of clusters at water activities higher than $40 \%$. The water diffusivity is almost independent of activity below $50 \% \mathrm{HR}$ and decreases rapidly when activity increases above $50 \%$. Contrary to CTH experiments, equilibrium is reached in DVS and the difference is not simply linked to the well-known effect of sample thickness on diffusion rate. The results allows hypotheses such as hydrolysis or osmotic cracking to explain the abnormal sorption phenomenon to be rejected. It is suggested that clusters could be polymer-water complexes having a linear / branched structure able to grow without phase separation that could explain the reversibility of sorption-desorption cycles. The difference of behavior between thin $0.1 \mathrm{~mm}$ and thicker 1.9 or $3.8 \mathrm{~mm}$ samples could be due to an effect of swelling stresses.
\end{abstract}

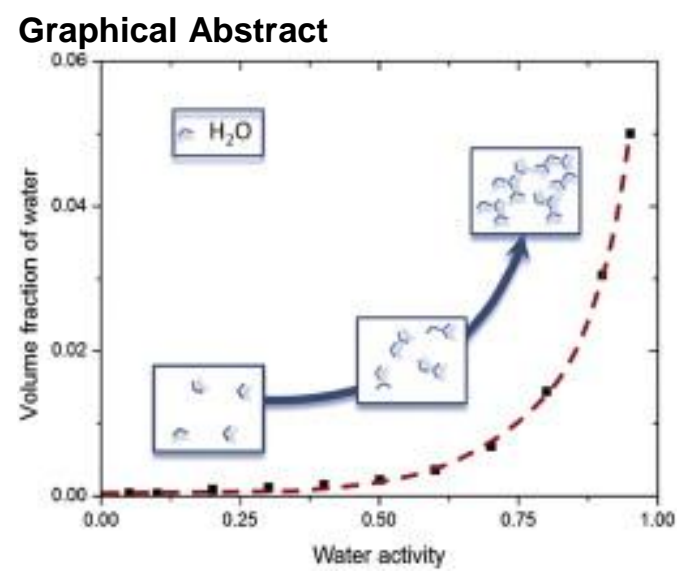

Keywords : polychloroprene ; water sorption ; clustering 


\section{Introduction}

Elastomers are generally polymers of low polarity, a condition to have a low cohesive energy density and thus a low glass transition temperature. Since water interacts with polymers through hydrogen bonds with polar groups it is expected to have a relatively low solubility in elastomers as is generally observed. But low solubility is not necessarily synonym of a simple dissolution mechanism. It was observed 50 years ago that in many elastomers, the equilibrium water volume fraction $v$ varies nonlinearly with water activity $a$ while the water diffusivity appears as a decreasing function of $a$ [1]. Both characteristics are generally attributed to clustering [2 - 5]. As will be shown, this term covers a variety of processes having in common the fact that a part of the water molecules establishes preferentially bonds with previously solvated water molecules rather than with polar sites of the polymer. The most trivial cause of clustering is the presence of pores in the elastomer matrix. When these pores are small enough, water condenses in the pores at water activities lower than unity owing to confinement effects and the sorption isotherm displays a positive curvature in the domain of high activities. Adsorption at the pore surface can have the same consequences as condensation. In the case under study, porosity can be considered negligible; water is first dissolved into the superficial layer of the elastomer but tends to adopt an inhomogeneous spatial distribution characterized by the existence of water-water bonds when, at the concentrations under consideration, each water molecule would be isolated from all the others in the case of an homogeneous distribution. One can envisage at least two mechanisms of clustering in non-porous polymers. The first one involves the presence of an extrinsic driving force, for instance a thermal shock inducing a sudden decrease of the water solubility in the polymer or the release of small hydrophilic organic molecules resulting for instance from matrix degradation. In such cases, a demixing process occurs; liquid micro-pockets are formed, creating a situation favorable for the propagation of an osmotic cracking process. Blistering of composite boat hulls, for instance, results from polyester hydrolysis $[6,7]$.

The second mechanism does not need the existence of a driving force, clustering can be schematically explained by the fact that water molecules have more affinity for themselves than for polymer sites. Here, clusters can be chains or networks $[8,9]$ of hydrogen bonded water molecules rather than quasi-spherical aggregates and it is not unreasonable to imagine relatively large clusters not phase separated from the polymer matrix contrary to quasi-spherical clusters.

The present article deals with water absorption mechanisms of vulcanized chloroprene in the $25^{\circ} \mathrm{C}$ $80^{\circ} \mathrm{C}$ temperature range. Long term aging experiments at constant temperature and hygrometric 
ratio will be combined with short term measurements in order to record sorption isotherms. A peculiar attention will be paid to clustering processes. 


\section{Experimental}

\section{Material}

The sample considered here is a vulcanized polychloroprene based on raw material with a weight average molar mass, assessed by GPC, close to $140 \mathrm{~kg} / \mathrm{mol}$. Vulcanization was performed by an industrial partner with both $\mathrm{ZnO}$ (5.7phr) and Sulphur (1.2phr) with no reinforcing fillers. Polychloroprene rubber, with a crosslink density of $0.18 \mathrm{~mol} / \mathrm{kg}$, was prepared in 1.8 and $3.8 \mathrm{~mm}$ thick sheets. A Leica microtome was used to cut 100 microns thick films from the 3.9 thick sheets after cooling in liquid nitrogen. After cutting, film quality has been checked in order to be sure that the material was not damaged before testing.

\section{Water Absorption}

Dynamic vapor sorption (DVS)

Dynamic Vapor Sorption measurements were performed with TA Instruments (Q5000SA) equipment to characterize water absorption in $0.1 \mathrm{~mm}$ thick films. This allows water uptake to be monitored using a microbalance with a $0.1 \mu \mathrm{g}$ resolution placed in a humidity chamber that can be controlled in both temperature (from 5 to $80^{\circ} \mathrm{C}$ ) and humidity (from 0 to $95 \%$ ).

The DVS measurements were made at $40^{\circ} \mathrm{C}$. After a drying period until the sample reaches equilibrium, water activity in the chamber is increased step by step using a flow of nitrogen-dry water vapor mixture at $200 \mathrm{~mL} / \mathrm{min}$. Mass evolution $m(t)$ is recorded with a frequency of about 0.1 $\mathrm{Hz}$ and translated into mass percent of absorbed water mass $M(t)$ :

$$
M(t)=\frac{m(t)-m_{0}}{m_{0}} * 100
$$

Where $m(t)$ is sample mass at time $t$ and $m_{0}$ is the mass of the dry polymer sample. The drying process was performed in situ by setting humidity level to 0 .

Constant Temperature and Hygrometry Experiment (CTH)

Samples were immersed in natural renewed sea water at different temperatures from $25^{\circ} \mathrm{C}$ to $80^{\circ} \mathrm{C}$. Sea water can be considered, here, as pure water with an activity of 0.98 according to Robinson [10]. The water absorption was determined from the weight evolution of square samples 
$(50 \mathrm{~mm} * 50 \mathrm{~mm})$ with two different thicknesses $(1.8$ and $3.9 \mathrm{~mm})$. Mass gain was followed by periodic weighing on a Sartorius LA $310 \mathrm{~S}$ balance (precision $0.1 \mathrm{mg}$ ). Samples were removed from the ageing containers and wiped with paper towels to dry the surfaces before weighing. The mass percent of absorbed water $M(t)$ of each sample at time $t$ is expressed using equation 1 . For each condition 3 samples were tested and results averaged.

\section{$\mathrm{HCl}$ measurement}

In order to investigate the possible release of $\mathrm{HCl}$ due to hydolysis of $\mathrm{C}-\mathrm{Cl}$ bonds, $1.8 \mathrm{~mm}$ thick samples of $\mathrm{CR}$ (cut in small squares) were aged in a small bottle $(100 \mathrm{~mL})$ of pure water at $95^{\circ} \mathrm{C}$ for more than 200 hours. After ageing, silver nitrate was used to reveal the presence of $\mathrm{HCl}$ in the water bath. For these experimental conditions, the measurement sensitivity was lower than $10^{-4} \mathrm{~mol}^{-\mathrm{L}^{-1}}$ meaning that the lowest rate of $\mathrm{HCl}$ formation that could be detected was $1.210^{-5}$. 


\section{Results}

The curves of mass gain against exposure time at constant temperature and hygrometry (CTH) are presented in Fig.1 for samples of $1.9 \mathrm{~mm}$ thickness and in Fig. 2 for samples of $3.8 \mathrm{~mm}$ thickness. These curves display a negative curvature in their initial part and a quasi-linear asymptote of which the slope is an increasing function of temperature and seems almost independent of sample thickness. The asymptotic rate values $r_{S}=\frac{1}{m_{0}}\left(\frac{d m}{d t}\right)_{\infty}$ are listed in Table 1 . An Arrhenius plot of $r_{s}$ values for both sample thicknesses is given in Figure 3 . At first sight, these plots appear non-linear, that would indicate the coexistence of at least two mechanisms. Indeed, the number of points is very small but the close similarity of both curves does not result from a coincidence and suggests that effectively, Arrhenius law is not obeyed here.

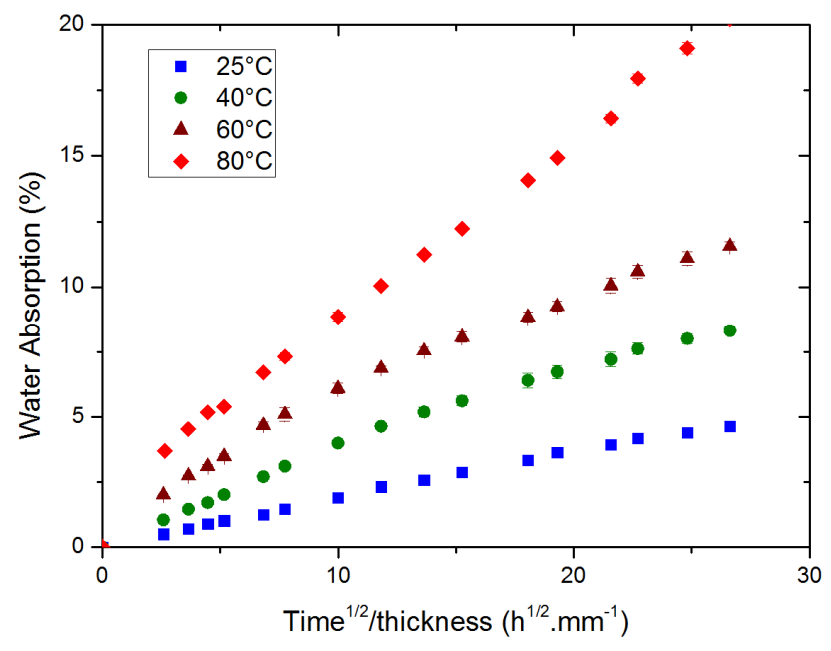

Figure 1 : Water absorption in $1.9 \mathrm{~mm}$ thick sample of $\mathrm{CR}$ at different temperatures.

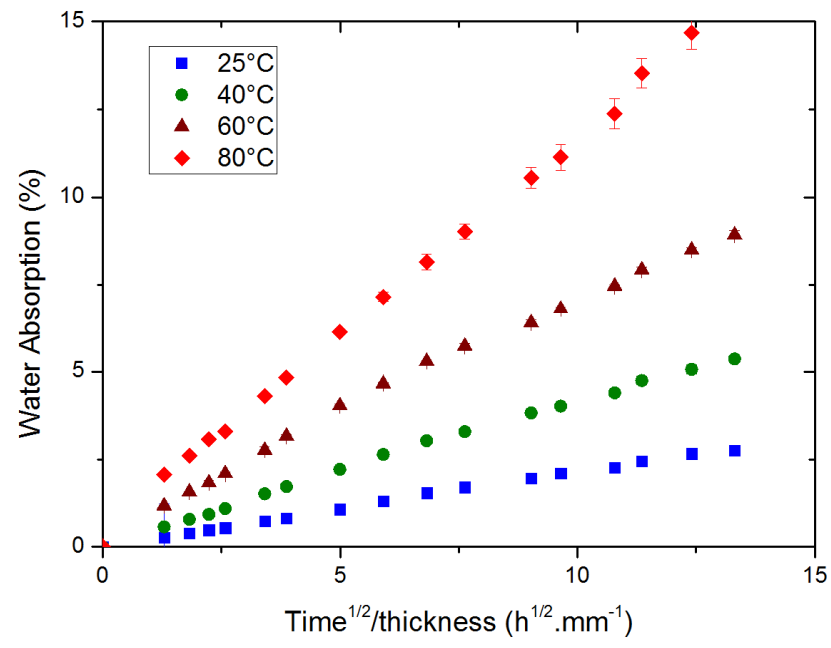

Figure 2 : Water absorption in 3.8mm thick sample of CR at different temperatures. 


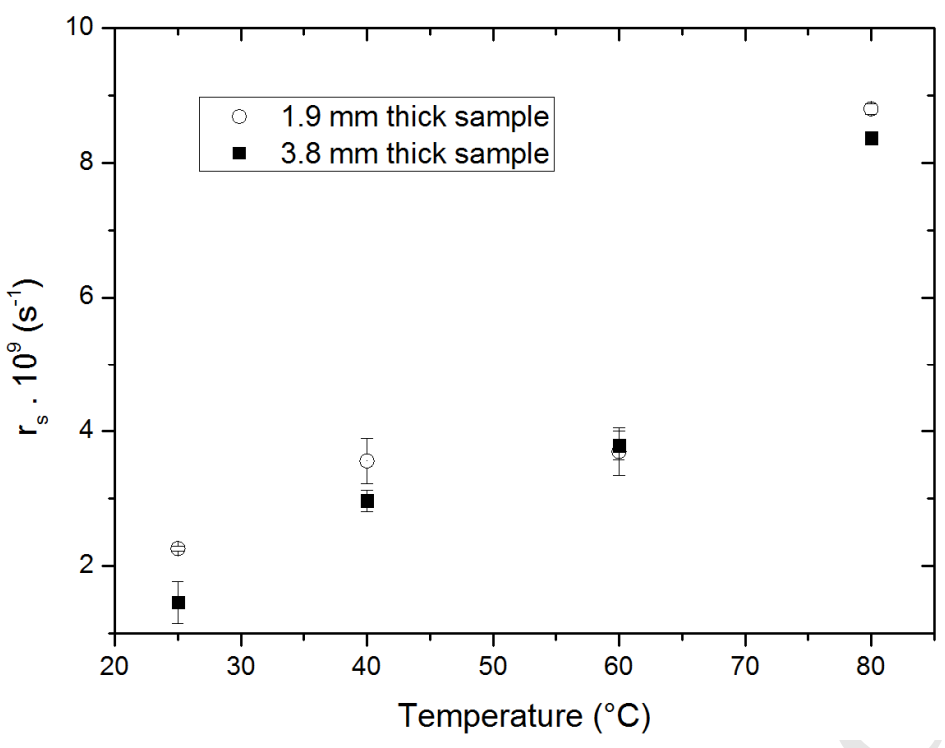

Figure 3 : Temperature dependence of $r_{s}$ (values given in Table 1).

The initial parts of the curves call for the following comments: At the lowest temperatures $25^{\circ} \mathrm{C}$ and $40^{\circ} \mathrm{C}$, mass gain increases proportionally to the square root of time in the first days of exposure. At the highest temperatures $60^{\circ} \mathrm{C}$ and $80^{\circ} \mathrm{C}$, mass gain is proportional to a power of time ranging between 0.5 and 1.0. It can be reasonably supposed that here also, there is an initial period where mass gain is proportional to the square root of time but this period is shorter than 24 hours. What is observed here is essentially the transition between the regime characterized by an exponent value of 0.5 and the "steady state " regime characterized by an exponent of unity. We have determined a pseudo-initial slope $v_{24 h}=\frac{1}{m_{0}} \frac{\Delta m(24 h)}{h \cdot(24 \times 3600)^{1 / 2}}$ where $\mathrm{h}$ is sample thickness. In the case of the lowest temperatures $\left(25^{\circ} \mathrm{C}\right.$ and $\left.40^{\circ} \mathrm{C}\right), v_{24 h}$ is effectively the initial slope $v_{0}$. In the case of the highest temperatures $\left(60^{\circ} \mathrm{C}\right.$ and $\left.80^{\circ} \mathrm{C}\right)$, it can be supposed that $v_{0}>v_{24 h}$ but the difference is presumably small and one can consider, in a first approach, that $v_{24 h}$ is effectively the initial slope. Contrary to $r_{s}$, $v_{0}$ varies significantly with the sample thickness (Table 1 ), but the product $V_{0}=L \cdot v_{0}$ is almost independent of the thickness $L$. It can be recalled that, in the context of a fickian diffusion process, $V_{0}$ is an important component of the expression of the diffusion coefficient:

$D=\frac{\pi}{16} \frac{V_{0}^{2}}{\left(\Delta_{m \infty} / m_{0}\right)^{2}}$

The kinetic curves of mass gain can thus be considered as resulting from two processes: a (relatively fast) pseudo-fickian sorption process characterized by a virtual equilibrium mass gain $\Delta m_{\infty}$ (see below) and a (relatively slow) unknown process of apparent zero order, occurring at the same rate in all the sample layers.

Let us first consider the equation of the linear asymptote: 
$\frac{\Delta m}{m_{0}}=r_{s} t+b$

The values of $b$ are listed in Table 1 . This intercept value can be interpreted, at least in a first approach, as the (virtual) equilibrium value for the initial fickian process:

$b=\frac{\Delta m_{\infty}}{m_{0}}$

From the values of $V_{0}$ and $b$, it is possible to determine the value of the coefficient of diffusion $D$ using Equ.4 (Table 1). D appears to be about three times higher for the sample of $3.8 \mathrm{~mm}$ thickness than for the sample of $1.9 \mathrm{~mm}$. This is exclusively due to the difference in b values, which increase almost linearly with temperature:

$b=\frac{\Delta m_{\infty}}{m_{0}} \sim A(T-13)$

Where $A \sim 2.0 .10^{-3} \mathrm{~K}^{-1}$ for samples of $1.9 \mathrm{~mm}$ and $1.1 .10^{-3} \mathrm{~K}^{-1}$ for samples of $3.8 \mathrm{~mm}$. As will be seen below, the physical meaning of this dependence is not obvious. What is sure is that the values of $\frac{\Delta m_{\infty}}{m_{0}}$ do not correspond to the true water solubility in polychloroprene, this latter being considerably lower.

\begin{tabular}{|l|l|l|l|l|l|l|}
\hline $\begin{array}{l}\text { Sample thickness } \\
(\mathrm{mm})\end{array}$ & $\begin{array}{l}\text { Temperature } \\
\left({ }^{\circ} \mathrm{C}\right)\end{array}$ & $\begin{array}{l}\mathrm{r}_{\mathrm{s}} \times 10^{9} \\
\left(\mathrm{~s}^{-1}\right)\end{array}$ & $\begin{array}{l}\mathrm{b} \times 10^{2} \\
\mathrm{bxL}\end{array}$ & $\begin{array}{l}\mathrm{v}_{0} \times 10^{8} \\
(\mathrm{~mm})\end{array}$ & $\begin{array}{l}\mathrm{D} \times 10^{13} \\
\left(\mathrm{~m}^{-1 / 2}\right)\end{array}$ & $\left.\mathrm{m}^{2} . \mathrm{s}^{-1}\right)$ \\
\hline 1.9 & 25 & $2.26+/-0.04$ & $2.67+/-0.17$ & $5.07+/-0.32$ & $3.23+/-0.17$ & $2.9+/-0.7$ \\
\hline 1.9 & 40 & $3.56+/-0.33$ & $5.23+/-0.44$ & $9.94+/-0.83$ & $6.77+/-0.44$ & $3.3+/-0.1$ \\
\hline 1.9 & 60 & $3.70+/-0.35$ & $8.13+/-0.47$ & $15.45+/-0.89$ & $13.00+/-0.19$ & $5.0+/-0.1$ \\
\hline 1.9 & 80 & $8.80+/-0.08$ & $12.07+/-0.53$ & $22.93+/-1.01$ & $23.83+/-0.50$ & $7.7+/-0.1$ \\
\hline 3.8 & 25 & $1.46+/-0.31$ & $1.47+/-0.05$ & $5.58+/-0.19$ & $3.48+/-0.09$ & $11.0+/-1.3$ \\
\hline 3.8 & 40 & $2.97+/-0.16$ & $2.74+/-0.15$ & $10.41+/-0.57$ & $7.41+/-0.08$ & $14.4+/-1.8$ \\
\hline 3.8 & 60 & $3.79+/-0.21$ & $5.46+/-0.08$ & $20.75+/-0.30$ & $15.20+/-0.07$ & $15.2+/-0.6$ \\
\hline 3.8 & 80 & $8.37+/-0.05$ & $7.95+/-0.11$ & $30.21+/-0.41$ & $26.70+/-0.54$ & $22.1+/-1.5$ \\
\hline
\end{tabular}

Table 1. Kinetic parameters of water absorption (see text) in CTH experiments

In order to provide more information on this abnormal sorption process, we have first performed cyclic tests in which the samples are maintained at constant temperature and periodically dried after exposure in humid atmosphere. The results (Fig.4) can be summarized as follows: water absorption is fully reversible and there is no significant change of the diffusion coefficient from one cycle to the next. The fact that $D$ remains constant indicates the absence of irreversible damage resulting for instance from osmotic cracking. If there is a polymer-water phase separation, we are forced to 
imagine that the water pockets become voids upon drying, these latter would then collapse and heal in such a way that the polymer homogeneity was restored at the end of the drying period. But what could be the driving force of phase separation? Osmotic cracking would need the presence of small polar molecules soluble in water but having diffusivity values significantly lower than that of the water in the polymer. We have imagined that hydrolysable $\mathrm{C}-\mathrm{Cl}$ bonds could be present in irregular structures and that the released $\mathrm{HCl}$ would induce osmotic cracking. An experiment of sample immersion in a bath of pure boiling water was therefore performed, but no $\mathrm{HCl}$ was found in the bath after an exposure time of 200 hours, which eliminates the hypothesis of hydrolysis of labile $\mathrm{C}-\mathrm{Cl}$ bonds.

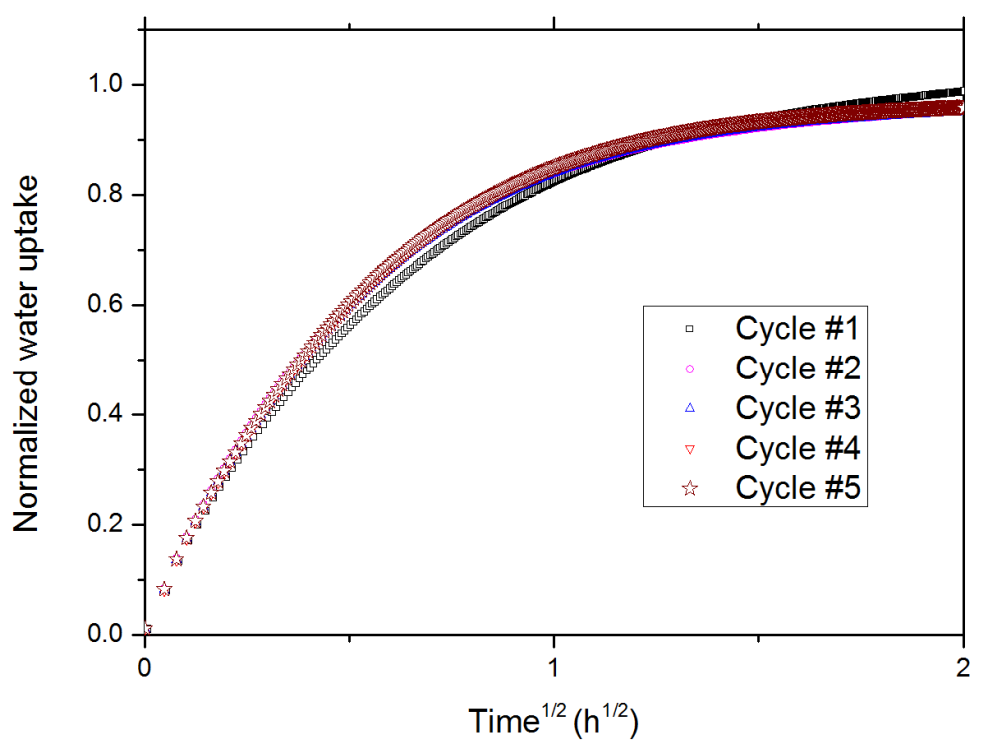

Figure 4 : Cyclic tests of water absorption in CR

Polymer hydrolysis could explain the observed behavior, provided that water molecules are incorporated in the polymer structure without mass loss. It can be noted that mass gain reaches values higher than $20 \%$ in $1.9 \mathrm{~mm}$ samples and that corresponds to one mole of water per mole of monomer unit. The only possible hydrolysis event would be thus the addition of the water molecule to the double bond but that is unlikely, at least in the temperature range under consideration. The hypothesis of hydrolysis to explain the abnormal mass gain must thus also be rejected.

At the end of this set of investigations, it has been shown that the mechanism of water absorption responsible for the continuous mass increase at long term is neither hydrolysis nor osmotic cracking, it is fully reversible and able to incorporate in polychloroprene more than one water molecule per monomer unit. The questions which remain unanswered lead us to investigate sorption mechanisms 
at the molecular level. The analysis of sorption isotherms could bring interesting information on this aspect; for this reason Dynamic Vapor Sorption (DVS) experiments were performed.

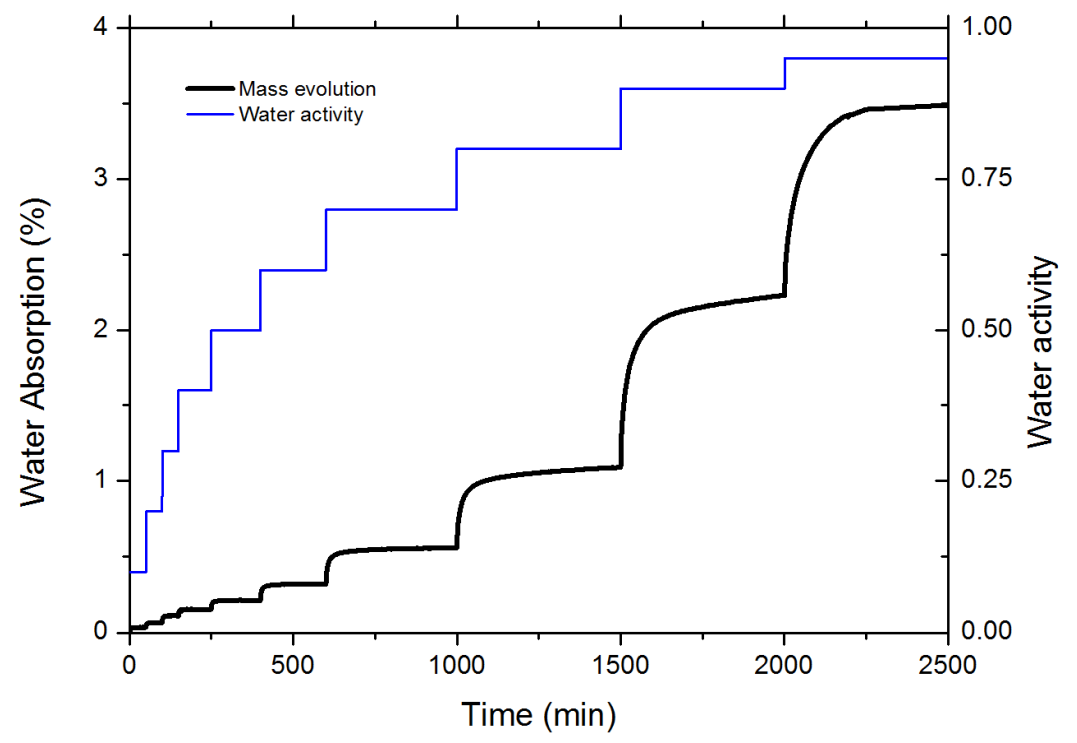

Figure 5 : Water absorption in $100 \mu \mathrm{m}$ thick film for several water activities at $40^{\circ} \mathrm{C}$.

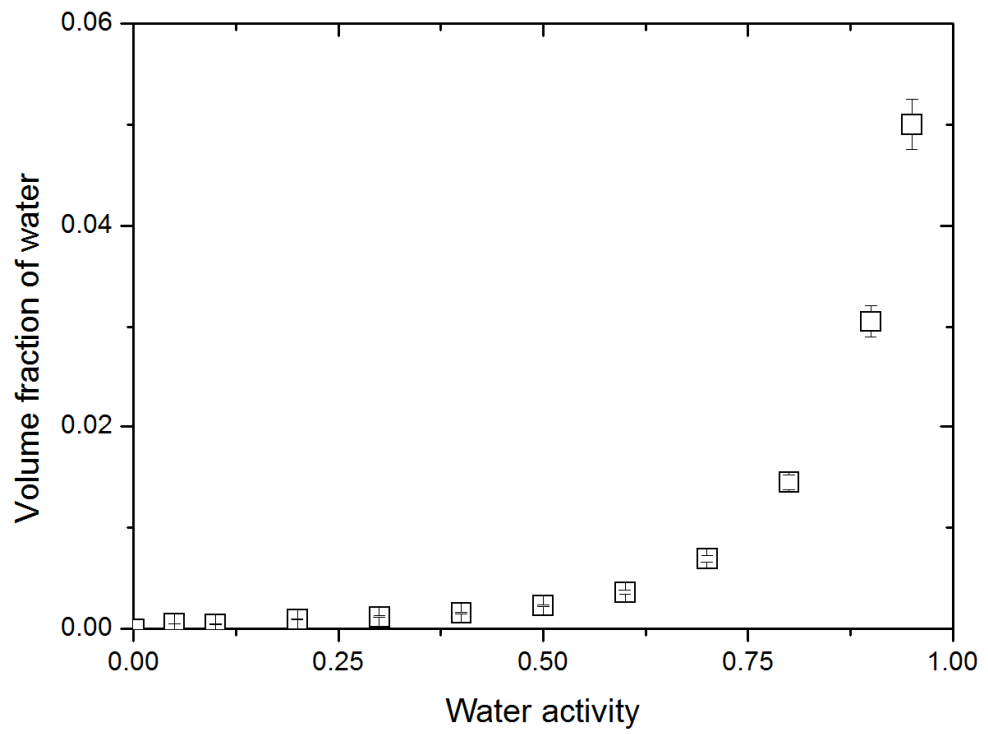

Figure 6 : Volume fraction of water in $100 \mu \mathrm{m} \mathrm{CR}$ film at $40^{\circ} \mathrm{C}$ for different water activities.

A typical sorption curve is shown in Fig. 5 and the sorption isotherm obtained at $40^{\circ} \mathrm{C}$ on a sample of 100 micrometers thickness, is shown in Fig.6. The most striking difference between this curve and those of Figs 1 and 2 is that equilibrium is reached in less than 10 hours while it is not reached after more than 2500 hours during the experiment at constant temperature and hygrometry (CTH). Within 
a fickian diffusion mechanism, the time to reach equilibrium would be proportional to the square of thickness so that equilibrium would be reached in less than $19^{2}=361$ hours for the sample of $1.9 \mathrm{~mm}$ and $38^{2}=1444$ hours for the sample of $3.8 \mathrm{~mm}$ thickness. It is clear that the effect of sample thickness on the sorption behavior cannot be simply derived from Fick's law.

The sorption isotherm displays a strong positive curvature in the domain of high activities. There are to our knowledge, two main ways to interpret this behavior. In the first, the relationship between the water volume fraction $u$ and water activity $a$ would obey the well-known Flory-Huggins equation; in the second one, water dissolution would obey Henry's law and the isotherm curvature would be explained by clustering.

Let us consider the first hypothesis. By linear regression on the earlier points of the isotherm, one can determine the initial slope $H$ :

$H=\left(\frac{d u}{d a}\right)_{a=0}=4.6 .10^{-3} \quad\left(R^{2}=0.956\right)$

According to the Flory-Huggins equation:

$H=\exp -(1+\chi)$

Where $\chi$ is the polymer-water interaction coefficient. Application of this equation would give $\chi=4.38$. For such a value, the Flory-Huggins isotherm would be almost linear over the whole activity scale and the water volume fraction at saturation would be close to $4.6 \cdot 10^{-3}$ compared to the experimental value of $71.10^{-3}$. It is thus clear that the isotherm curvature does not correspond to the Flory-Huggins equilibrium.

Let us now examine the second hypothesis. The isotherm shape can be then analyzed with the ZimmLundberg theory [11]. These authors define a clustering function $f_{Z L}$ defined by:

$$
f_{Z L}=-(1-u)\left(\frac{\partial\left(\frac{a}{u}\right)}{\partial a}\right)-1
$$

Clusters are present when $f_{Z L}>-1$. The average cluster size I is given by:

$$
l=u f_{Z L}+1
$$

The isotherms can be fitted by the sum of a Henry's term and a power term representing the cluster contribution, in order to have an analytical representation of isotherms facilitating calculations [12]:

$u=H a+B a^{n}$ 
$B$ and $n$ were determined from experimental results. The regression analysis leads to $B=67.10^{-3}$ and $n$ $=9.1\left(R^{2}=0.999\right)$. This relationship leads to particularly simple expressions of Zimm-Lundberg quantities at saturation $\left(u=u_{s}=H+B\right.$ at $\left.a=1\right)$ :

$$
f_{Z L S}=B(m-1) \frac{\left(1-u_{s}\right)}{u_{s}^{2}}-1 \text { and } l_{s}=\left(1-u_{s}\right)\left[\frac{B(m-1)}{u_{s}}+1\right]
$$

The numerical application lead to $l_{s}=7.1$.

The coefficient of diffusion $D$ has been determined and plotted against hygrometric ratio $H R$ at $40^{\circ} \mathrm{C}$ in Fig.7. D is almost independent of HR between about 10 and 50\% HR and decreases rapidly with HR above $50 \% \mathrm{HR}$. The $\mathrm{D}$ value at $100 \% \mathrm{HR}$ (about $3.10^{-13} \mathrm{~m}^{2} \cdot \mathrm{s}^{-1}$ ) is not very different from the one obtained in CTH experiment for the sample of $1.9 \mathrm{~mm}$ thickness.

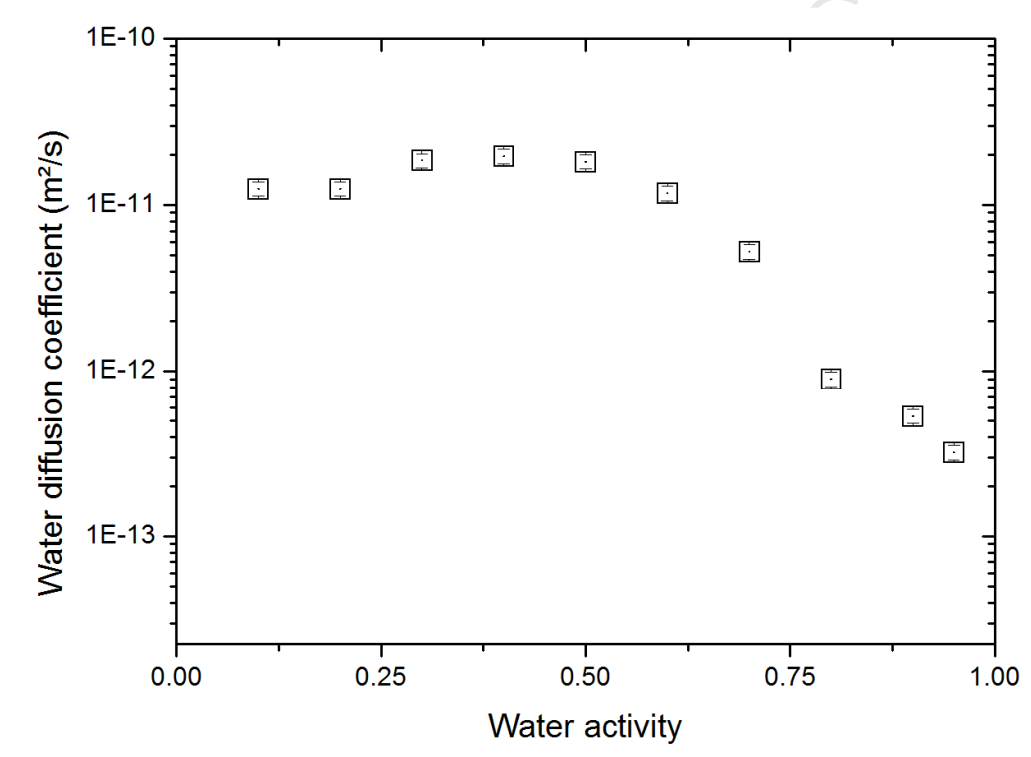

Figure 7 :Water diffusion coefficient measured at $40^{\circ} \mathrm{C}$ on 100 micron film for different water activities

To conclude on DVS results: polychloroprene is characterized by a very low water solubility (obeying Henry's law with a dimensionless solubility coefficient $\mathrm{H}=4 \cdot 6 \cdot 10^{-3}$ ). But it is also characterized by the formation of clusters at moderate-high activities, to which the Zimm-Lundberg theory applies. Since the hypothesis of osmosis has been rejected, we have to find a proposal for another clustering mechanism. At high water activity values, the clusters slow down the diffusion rate as previously observed [2-4], the diffusion coefficient being about 30 times lower at $100 \% \mathrm{HR}$ than at $10-20 \% \mathrm{HR}$.

It is possible to envisage clustering from the (purely physical) formation of polymer-water complexes involving several water molecules. Water clustering occurs frequently in polymers of low polarity 
because water molecules bound to the polymer compete efficiently with polymer polar sites to "capture" new water molecules. An interesting theory of clustering was developed at the beginning of the 1960's $[13,14]$, it considers that a water molecule is able to establish 4 hydrogen bonds and then behaves as a tetrafunctional monomer of type $A_{4}$ forming a network. Gelation would then correspond to saturation and the Flory's theory for network formation would be applicable. An important parameter in this theory is the equilibrium constant for the formation-dissociation of hydrogen bonds. Clustering conditions can be illustrated by a simpler model in which cluster growth is limited to two water molecules:
$P+W--->P W$
$\left(\mathrm{k}_{\mathrm{c} 1}\right)$
PW ------> P+W
$\left(k_{\mathrm{d} 1}\right)$
PW + W ----> PW2
$\left(k_{c 2}\right)$
PW2 -----> PW + W
$\left(k_{d 2}\right)$

Where $\mathrm{P}$ is a polymer polar site, $\mathrm{W}$ is the water molecule, $[\mathrm{W}]$ is the concentration of non-bonded water in the polymer, presumably proportional to water activity. PW and PW2 are polymer-water complexes with respectively one and two water molecules; $k_{c 1}$ and $k_{c 2}$ are (second order) rate constants for complex formation; $k_{\mathrm{d} 1}$ and $k_{\mathrm{d} 2}$ are (first order) rate constants for complex decomposition. The number $\mathrm{N}$ of bound water molecules is given by:

$N=[P W]+2[P W 2]$

The complex concentration varies according to the following kinetic equations:

$$
\begin{aligned}
& \frac{d[P W]}{d t}=k_{c 1}[P][W]-k_{d 1}[P W]-k_{c 2}[P W][W] \\
& \frac{d[P W 2]}{d t}=k_{c 2}[P W][W]-k_{d 2}[P W 2]
\end{aligned}
$$

In the stationary state $\mathrm{N}$ is constant so that:

$$
\frac{d[P W]}{d t}+2 \frac{d[P W 2]}{d t}=0
$$

The equilibrium cluster (PW2) concentration is then:

$$
[P W 2]_{\infty}=\frac{k_{c 1} k_{c 2}[W]^{2}[P]}{3 k_{c 2} k_{d 2}[W]-k_{d 1} k_{d 2}}
$$

Clustering exists only if [PW2] $>0$ i.e. if: 
$3 k_{c 2}[W]>k_{d 1}$

Two conditions appear, the first one on rate constants, $[\mathrm{W}]_{s}$ being the water concentration at saturation:

$$
R_{1}=\frac{3 k_{c 2}[W]_{s}}{k_{d 1}}>1
$$

If this condition was not obeyed, no clustering would occur whatever the water activity.

The second one illustrates the existence of a critical water concentration $[\mathrm{W}]_{c}$, therefore a critical water activity $a_{c}$ below which no cluster is formed:

$$
[W]>[W]_{c}=\frac{k_{d 1}}{3 k_{c 2}}
$$

With our analytical model of sorption isotherms, it is effectively possible to define arbitrarily a critical water activity $a_{c}$ such that the ratio power term/Henry's term is equal to the relative uncertainty on water volume fraction (or mass uptake):

$$
\frac{B a_{c}^{n}}{H a_{c}}=\frac{\Delta u}{u}
$$

Taking arbitrarily a relative uncertainty of $1 \%$ one obtains $a_{c} \sim 0.41$.

Indeed the above model is an oversimplification. However it is difficult for us to imagine another configuration than the one consisting of the presence of clusters with a certain size distribution determined by their stability i.e; their ability to decompose by releasing successively water molecules or by splitting into smaller units. Among the infinite diversity of possible configurations, the above model describes the simplest one but it differs only qualitatively from a more complex model of the same type. It is not proposed here as the definitive solution of the problem but rather as an interesting point of departure towards more realistic models. The most difficult problem here will not be to find a good solution; it is rather to demonstrate that it is that there is no other solution. Two important points should be considered here:

First about the existence or not of a phase separation: if the clusters were almost spherical, centered on a polar site of the polymer, it would be difficult to imagine the formation of large clusters without phase separation, and the existence of this latter without irreversible damage. But in fact, PWn clusters can be linear, eventually branched, as found by molecular dynamics simulation [9] but also by dielectric or NMR measurements $[15,16]$, and can reach relatively large sizes without phase separation, which could solve the problem of full reversibility.

Second concerning equilibrium, DVS results show that an apparent equilibrium is established rapidly i.e. that the rate constants $k_{c j}$ and $k_{d i}$ have relatively high values. In this case, the explanation could be 
linked to the high water concentration reached in samples at high activities: more than $15 \%$, predominantly in clusters. A rubber sample swelled by a large quantity of clustered water is no doubt a fragile object in De Genne's sense [17], i.e. it is expected to undergo significant changes under small stimuli, for instance linked to swelling stresses. This stress-water absorption coupling could explain the observed effect of sample thickness, for instance the slow increase of water concentration at almost constant rate could be linked to polymer creep under swelling stresses. This aspect has been investigated many years ago, for instance Crank [18] found that swelling stresses could explain an increase of water diffusivity with the sample thickness, as observed here (Table 1). However some aspects observed here, for instance the continuous mass increase at constant rate do not appear in Crank's work and will need further study.

\section{Conclusion}


The water absorption by polychloroprene has been studied at various temperatures and constant hygrometric ratios (CTH experiments) for long times (up to 2500 hours) on thick samples (1.9 and 3.8 $\mathrm{mm}$ ). It has also been examined at fixed temperature $\left(40^{\circ} \mathrm{C}\right)$ and various water activities (DVS experiments) for short times (a few hours) on thin samples $(100 \mu \mathrm{m})$. In CTH experiments, water absorption begins as a fickian process but does not display equilibrium, the water concentration continues increasing linearly with time when the diffusion process has apparently reached its equilibrium. It was demonstrated that this abnormal process is neither due to hydrolysis nor osmotic cracking. In DVS experiments, in contrast, equilibrium is apparently reached. The shape of the sorption isotherm plotted as the variation of water diffusivity with its activity clearly indicates the existence of a water clustering process, responsible for the major part of the absorbed water at high activities. For instance, at saturation, water mass uptake can reach values of the order of $15-20 \%$ by weight whereas water dissolution in the polymer contributes only for $0.46 \%$. Cyclic experiments have shown that mass changes are fully reversible, i.e. that the abnormal sorption process does not induce damage. There is no other clustering mechanism to explain the observed behavior than the formation of polymer-water complexes carrying several (seven on average) water molecules per polar site of the polymer. These complexes remain miscible to the polymer which explains the full reversibility of absorption curves in cyclic experiments. A possible way to explain the differences of behavior between thin and thick samples could be the existence of a relatively strong coupling between swelling stresses and water absorption. 


\section{References}

1) Barrie J.A. (1968) "Water in Polymers" in "Diffusion in polymers" J.Crank and G.S.Park eds. Academic Press London.

2) Barrie J.A., Machin D., Nunn A. (1975) Polymer 16, 811.

3) Schult K.A., Paul D.R. (1996) J. Polym. Sci. Part B Polymer Physics 34, 2805.

4) Yang D.K., Koros W.J., Hopfenberg H.B., Stannet V.T. (1985) J. Appl. Polym. Sci.30, 1035.

5) S. Naudy, F. Collette, F. Thominette, G. Gebel, E. Espuche (2014), J. Membr. Sci. 451, 293

6) Ashbee K.H.G., Franck F.L. Wyatt R.C. (1967) Proc. Roy. Soc. London A300, 415.

7) Gautier L., Mortaigne B., Bellenger V., Verdu J. (1999) Polymer 41, 2481.

8) Mijovic J., Zhang H. (2003) Macromolecules 36, 1279.

9) Marque G., Neyertz S., Verdu J., Prunier V., Brown D.(2008) Macromolecules 41, 3349.

10) R. A. Robinson (1954). The vapour pressure and osmotic equivalence of sea water. Journal of the Marine Biological Association of the United Kingdom, 33, pp 449-455.

11) Zimm B., Lundberg J.L. (1956) J. Phys. Chem. 60, 425.

12) Gaudichet-Maurin E. (2005) PhD thesis "Carcterisation et vieillissement d'une membrane d'ultrafiltration d'eau" Ecole Nationale Supérieure d'Arts et Métiers. Paris. (http://pastel.archives-ouvertes.fr/docs/00/50/01/00/PDF/These-Gaudichet-Maurin.pdf)

13) Gordon M., Hope C.S., Loan L.D., Roe R.J. (1960) Proc. Roy. Soc. London A258, 215.

14) Barrie J.A., Platt B. (1963) J. Polym. Sci. 4, 303.

15) Xu G., Gryte C.C., Nowick A.S., Li S.Z., Pak Y.S., Greenbaum S.G. (1989) J. Appl. Phys.66, 5290.

16) Lim B.S., Nowick A.S., Lee K.W., Viehbeck A. (1993) J. Polym. Sci. Part B Polym. Phys. 31, 545.

17) De Gennes (1992) Reviews of Modern Physics, Vol. 64, No. 3.

18) Crank J. (1956) «The Mathematics of Diffusion » Oxford University Press London. 